\title{
Application of the Somatosensory Interaction Technology Combined with Virtual Reality Technology on Upper Limbs Function in Cerebrovascular Disease Patients
}

\author{
Wangxiang Mai ${ }^{1}$, Liang Fang ${ }^{2}$, Zhuoming Chen ${ }^{1}$, Xiuping Wang², Wanting Li' ${ }^{1}$, Weiyi He ${ }^{3}$ \\ ${ }^{1}$ Department of Rehabilitation Medicine, The First Affiliated Hospital of Jinan University, Guangzhou, China; \\ ${ }^{2}$ Haizhu District Hospital of Traditional Chinese Medicine, Guangzhou, China; ${ }^{3}$ Department of Pediatrics, The First \\ Affiliated Hospital of Jinan University, Guangzhou, China
}

Correspondence to: Weiyi He, edch@163.com

Keywords: Cerebrovascular Disease, Somatosensory Interaction, Virtual Reality, Upper Limbs Function, Rehabilitation

Received: April 17, $2020 \quad$ Accepted: May 17, $2020 \quad$ Published: May 20, 2020

Copyright $\odot 2020$ by author(s) and Scientific Research Publishing Inc.

This work is licensed under the Creative Commons Attribution International License (CC BY 4.0).

http://creativecommons.org/licenses/by/4.0/

\section{(c) (1) Open Access}

\section{ABSTRACT}

Objective: To explore the effects of the somatosensory interaction technology combined with virtual reality technology on upper limbs function and activities of daily living (ADL) in cerebrovascular disease patients. Methods: Form January, 2019 to December, 2019, 80 cerebrovascular disease patients were recruited, and had been divided into control group ( $\mathrm{n}$ $=40)$ and observation group $(n=40)$, randomly. The control groups received conventional rehabilitation treatment, for 40 minutes per day, while observation group received conventional rehabilitation treatment, for 20 minutes per day, and virtual reality technology treatment, 20 minutes per day, 5 days a week for 4 weeks. Wolf Motor Function Test (WMFT), Fugl-Meyer Assessment-Upper Extremities (FMA-UE) and modified Barthel in$\operatorname{dex}(\mathrm{MBI})$ were used to assess the motor function of the upper limbs and ADL before and after treatment. Results: Before treatment, the scores of WMFT, FMA-UE and MBI were no significant difference between two groups $(P>0.05)$. The scores improved in both groups after treatment $(P<0.01)$, and were higher in the observation group than in the control group $(P<0.05)$. Conclusion: The somatosensory interaction technology combined with virtual reality technology could facilitate to improve the upper limbs function and ADL in cerebrovascular disease patients.

\section{INTRODUCTION}

Globally, about 15 million people suffer from cerebrovascular disease each year [1]. Cerebrovascular 
disease is a leading cause of death and long-term disability worldwide [2]. Cerebrovascular disease refers to the cerebral dysfunction caused by cerebrovascular diseases. Cerebrovascular disease refers to the disease caused by vascular occlusion, vascular rupture, vascular wall damage or permeability change due to embolism and thrombosis. From the nature of the disease, it can be divided into ischemic cerebrovascular disease, hemorrhagic cerebrovascular disease. From the perspective of etiological classification, cerebral apoplexy and intracranial aneurysms are more common in adults, while intracranial vascular malformation and abnormal cerebral vascular network are more common in children and adolescents. It has been reported that about $80 \%$ of patients with cerebrovascular diseases are left with upper limb dysfunction, which affects their ability to take care of themselves in daily life [3]. Persistent upper limb dysfunction after cerebrovascular disease is one of the most challenging problems in rehabilitation [4]. Traditional exercise rehabilitation is carried out through physical therapy and/or occupational therapy. The traditional rehabilitation training is monotonous and boring, so many patients can not adhere to the treatment for a long time. Moreover, the emphasis on motion decomposition training fails to provide task-specific training. After the completion of subacute rehabilitation programs, many patients still exhibit significant upper limb dyskinesia. Therefore, it is necessary to develop alternative or complementary methods to maximize brain plasticity after cerebrovascular disease. It is of great significance to explore new rehabilitation techniques to alleviate the neurological dysfunction and improve the function of cerebrovascular patients.

Virtual Reality (VR) technology is a computer-generated simulation of the real world. Its goal is to create an immersive feeling. By giving visual and auditory stimuli, users can be immersed and immersed in a virtual environment [5]. Compared with traditional rehabilitation methods, virtual reality technology has the advantages of creating more realistic scenes, providing repetitive training for specific tasks, and having a stronger sense of participation. Virtual reality technology has been applied to the rehabilitation of cerebrovascular patients more and more. The application of Kinect motion-sensing interaction technology in virtual reality technology can replace many complicated traditional equipment and increase the portability and flexibility of training. Research at home and abroad suggests that Kinect motion-sensing interaction technology and virtual reality technology have certain effects in the rehabilitation of hemiplegia of cerebrovascular disease, which can improve the upper limb movement function and daily living ability of cerebrovascular disease patients [6,7]. This study intends to explore the effects of kinect-based motion-sensing interaction technology and virtual reality technology in the rehabilitation of upper limb motor dysfunction in patients with cerebrovascular disease.

\section{MATERIALS AND METHODS}

\subsection{General Information}

From January 2019 to December 2019, 80 patients with cerebrovascular diseases were admitted to Haizhu district hospital of traditional Chinese medicine in Guangzhou. The diagnosis all met the "cerebrovascular disease diagnostic criteria" of Chinese medical association, and were confirmed by head CT and/or MRI [8].

Inclusion criteria: 1) Age 8 - 65; Onset 3 weeks to 6 months, no consciousness disorders, good cognitive function (MMSE $\geq 22$ minutes), good coordination with the therapist; 2) Upper extremity Brunnstrom with stage III or above; Upper limb muscle strength grade 3 or above; Modified Ashworth scale $\leq$ level 2. 3) Patient balance function: the sitting balance reaches level 2. 4) The patient gave informed consent to the treatment and signed the treatment consent.

Exclusion criteria: 1) Obvious pain, dislocation of shoulder joint and limitation of movement in the affected upper limb. 2) Persons with severe mental disorder, severe cognitive impairment, language impairment, or dementia. 3) Patients with deteriorating condition, recurrent cerebral infarction or cerebral hemorrhage.

All the patients were divided into the control group and the treatment group according to the random number table method, with 40 cases in each group. There was no significant difference between the two groups in gender, age, disease course, lesion nature, hemiplegic side, etc. $(\mathrm{P}>0.05)$ (Table 1$)$. 
Table 1. The general data of the two groups were compared (n).

\begin{tabular}{|c|c|c|c|c|c|c|c|c|}
\hline \multirow{2}{*}{ Group } & \multicolumn{2}{|c|}{ Gender (n) } & \multirow[t]{2}{*}{ Age (years) } & \multirow[t]{2}{*}{$\begin{array}{l}\text { Course of } \\
\text { disease } \\
\text { (month) }\end{array}$} & \multicolumn{2}{|c|}{ Nature of lesions (n) } & \multicolumn{2}{|c|}{$\begin{array}{l}\text { Hemiplegic } \\
\text { side (n) }\end{array}$} \\
\hline & Male & Female & & & $\begin{array}{c}\text { Ischemic } \\
\text { cerebrovascular } \\
\text { disease }\end{array}$ & $\begin{array}{c}\text { Hemorrhagic } \\
\text { cerebrovascular } \\
\text { disease }\end{array}$ & $\begin{array}{l}\text { The } \\
\text { left } \\
\text { side }\end{array}$ & $\begin{array}{l}\text { The } \\
\text { right } \\
\text { side }\end{array}$ \\
\hline $\begin{array}{l}\text { The control } \\
\text { group }(\mathrm{n}=40)\end{array}$ & 19 & 21 & $48.45 \pm 6.28$ & $3.3 \pm 1.6$ & 23 & 17 & 25 & 24 \\
\hline $\begin{array}{l}\text { The treatment } \\
\text { group }(\mathrm{n}=40)\end{array}$ & 22 & 18 & $50.87 \pm 6.35$ & $3.2 \pm 1.8$ & 22 & 18 & 15 & 16 \\
\hline
\end{tabular}

\subsection{Treatment}

The control group only received traditional operation treatment, including: drum training, upper limb ball training, wood nail board training, screw training and other training. Bobath handshake training, the affected side of the upper limb support weight training, upper limb separation and control ability training, passive exercise training and other operational training. Train 40 minutes a day, 5 days a week for 4 weeks.

The treatment group received $20 \mathrm{~min}$ of traditional occupational therapy and 20 min of virtual reality game training based on Kinect motion-sensing interaction technology each time, 5 days a week for a total of 4 weeks. The virtual training program and difficulty are selected according to the patient's condition. Virtual reality game training system based on Kinect motion-sensing interaction technology consists of computer host and monitor, Kinect, projector, projection screen, audio equipment and protective facilities. This system mainly consists of cutting food and shooting games. Specific methods are as follows: 1) Cut food game training (Figure 1): take sitting or standing, the game began, when food appeared beating inside ark, with slowly raise the upper extremities, through concrete's shoulder, and elbow flexion and adduction, outreach and other composite action, move in different directions, knife to run the picture of food contained in the location (such as the top left, center, right, etc.), when at the same time satisfy the shoulder joint flexion, shoulder adduction or outreach, elbow extension, reach setting Angle, knife success "cut" food, the prompt tone of encourage sound, the target food disappeared. If the patient has limited shoulder and elbow movement or poor upper limb strength, the patient can be encouraged to assist the affected side with the healthy side to complete the training requirements. The console has "elbow flexion complementary Angle", "shoulder forward flexion Angle" and "upper limb horizontal adduction Angle". The bigger the three angles, the more difficult the training. 2) Shooting game training (Figure 2): take sitting or standing, slowly raise upper extremities, and shoulder forward bends, elbows straight as far as possible at the same time, the shooting target move, in the process, raise the upper limb "run" the picture of a gun, aim at the bull 'S-eye, when at the same time satisfy the shoulder joint flexion, elbow extension, gun shot a bullet hit the bull' S-eye, and hear the sound of the feedback. If the patient's elbow joint tension is high, can encourage the patient to use the healthy side to drive the affected side, complete the training requirements. There are two options on the console: "shoulder forward bend Angle" and "elbow extension Angle". Each time is 3 - 5 min, with 1 - 2 min rest. Each game can be set according to the patient's condition of different difficulty, to achieve individual treatment. At the end of each training session, the patient's performance was recorded. 

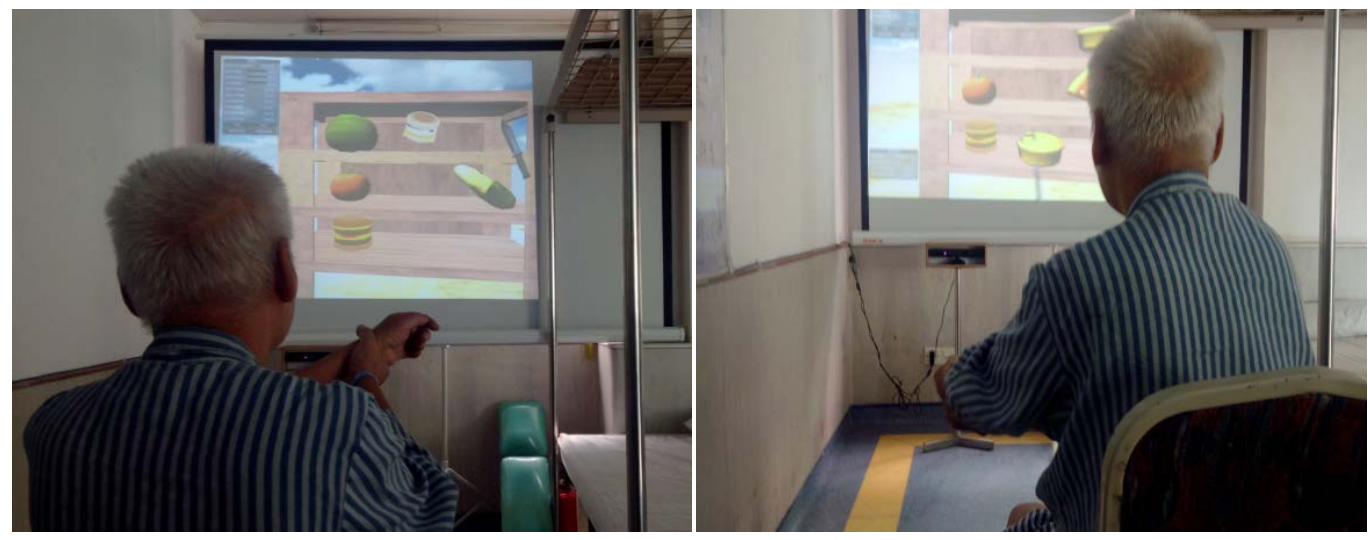

Figure 1. Participants were trained in VR task of cutting food.

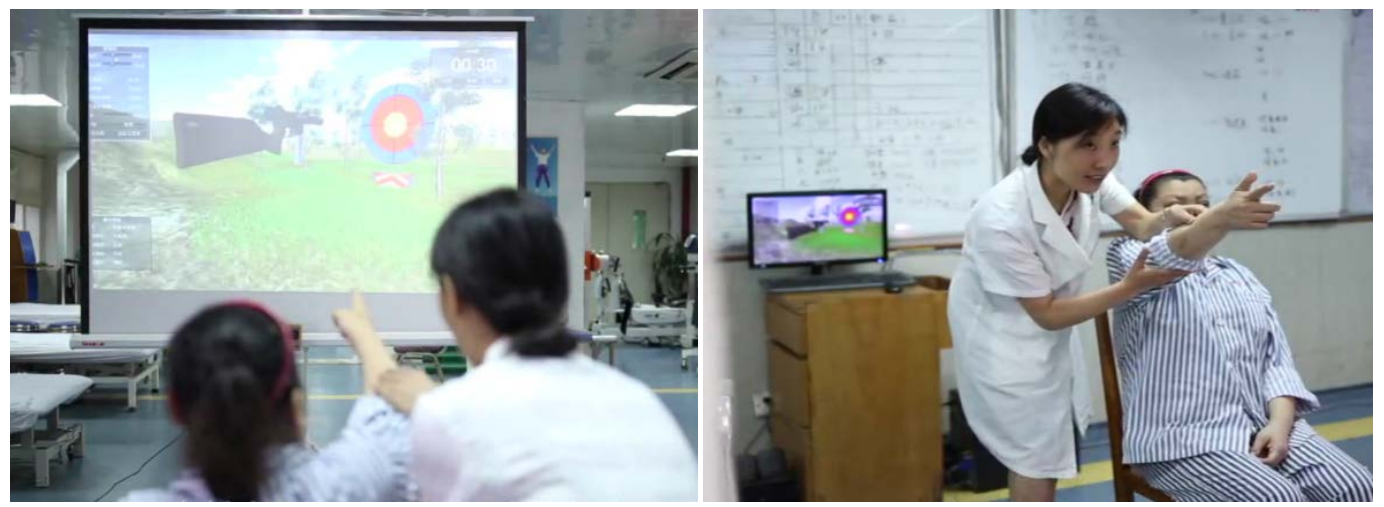

Figure 2. Participants undergo VR shooting task training.

\subsection{Evaluation Criteria}

1) The Wolf Motor Function Test (WMFT) was used to assess the upper limb motor function of the patients, and a rating scale was used to evaluate the upper limb motor function and dexterity. There were 15 rating items in total. Each item was rated as level 6,0-5 according to the degree of dysfunction. The full score is 75 points. The higher the score, the better the upper limb motor function.

2) Fugl-Meyer Assessment of Upper Extremities (FMA-UE) was assessment of patients with Upper limb movement function. A total of 33 evaluation items, full score of 66 points. The higher the score, the better the upper limb motor function.

3) The Modified Barhel Index (MBI) was used to assess the ability of patients to take care of themselves in daily life, out of 100 points.

Each patient was evaluated before and after treatment.

\subsection{Statistical Methods}

All data were input into Excel and analyzed by SPSS17.0 statistical software. The measurement data were expressed as mean \pm standard deviation. The t-test of paired data was used to conduct statistical comparison between the two groups before and after treatment, and the t-test of two independent samples was used to conduct statistical comparison between the two groups. $\mathrm{P}<0.05$ was considered statistically significant.

\section{RESULTS}

Before treatment, there was no significant difference in WMFT score, FMA-UE score and MBI score 
between the control group and the treatment group $(\mathrm{P}>0.05)$. After 4 weeks of treatment, WMFT score, FMA-UE and MBI score in the control group and the treatment group were significantly improved $(\mathrm{P}<$ 0.01 ), and the improvement in the treatment group was more significant than that in the control group ( $\mathrm{P}$ $<0.05)$ (Tables 2-4).

\section{DISCUSSION}

Virtual reality technology is increasingly used in the rehabilitation of cerebrovascular diseases [9]. Recent studies have shown that VR is an emerging treatment option for upper limb rehabilitation in stroke patients [10]. VR can provide users with an experience that simulates a "real" environment [11]. It provides a more diverse and realistic sensory experience for the subjects, and simulates the physical movement in daily life, making the rehabilitation more entertaining $[12,13]$. VR's gameplay quality can make patients feel fun in treatment and enhance their initiative and enthusiasm. Studies have shown that VR technology can effectively improve upper limb function in patients with cerebrovascular disease. Merians et al. combined robot technology with VR technology to design an upper limb assisted rehabilitation training system [14]. The results of rehabilitation training of 12 patients with sequelae stage of cerebrovascular disease indicated that the stability of the proximal limb of the affected side was improved, the smoothness and efficiency of movement were improved, and the upper limb function of the affected side was improved. Many VR systems, such as robotics, are expensive and must be treated under the guidance of medical staff. They are intended for use only in clinical settings and are not intended for remote or family rehabilitation. Somatosensory interaction technology refers to the human-computer interaction technology that interacts with the environment directly by means of body movement, voice, etc., and the device

Table 2. Comparison of WMFT scores before and after treatment between the two groups.

\begin{tabular}{ccccc}
\hline Group & Before the treatment & After treatment & $\mathrm{t}$ & $\mathrm{P}$ \\
\hline The control group $(\mathrm{n}=40)$ & $32.32 \pm 9.42$ & $40.55 \pm 11.07$ & -7.12 & 0.000 \\
The treatment group $(\mathrm{n}=40)$ & $30.77 \pm 8.17$ & $47.75 \pm 7.86$ & -18.45 & 0.000 \\
$\mathrm{t}$ & 0.78 & -3.35 & & \\
$\mathrm{P}$ & 0.43 & 0.001 & & \\
\hline
\end{tabular}

Table 3. Comparison of FMA-UE score before and after treatment between the two groups.

\begin{tabular}{ccccc}
\hline Group & Before the treatment & After treatment & $\mathrm{t}$ & $\mathrm{P}$ \\
\hline The control group $(\mathrm{n}=40)$ & $30.35 \pm 5.78$ & $38.92 \pm 5.26$ & -18.59 & 0.000 \\
The treatment group $(\mathrm{n}=40)$ & $28.72 \pm 4.79$ & $43.32 \pm 5.47$ & -15.98 & 0.000 \\
$\mathrm{t}$ & 1.36 & -3.664 & & \\
$\mathrm{P}$ & 0.175 & 0.000 & & \\
\hline
\end{tabular}

Table 4. Comparison of MBI scores before and after treatment between the two groups.

\begin{tabular}{ccccc}
\hline Group & Before the treatment & After treatment & $\mathrm{t}$ & $\mathrm{P}$ \\
\hline The control group $(\mathrm{n}=40)$ & $52.27 \pm 14.32$ & $62.67 \pm 13.52$ & -12.17 & 0.000 \\
The treatment group $(\mathrm{n}=40)$ & $50.15 \pm 13.53$ & $69.67 \pm 10.23$ & -14.68 & 0.000 \\
$\mathrm{t}$ & 0.68 & -2.61 & & \\
$\mathrm{P}$ & 0.49 & 0.011 & & \\
\hline
\end{tabular}


recognizes the user's movement and gives feedback. It features no mouse, keyboard or other controls, allowing users to interact more freely. The Kinect sensor is cheap and powerful, and is increasingly being used in the field of rehabilitation medicine. In recent years, Kinect has proved its role in the field of rehabilitation. Qu Chang et al. developed an upper limb rehabilitation training system based on Kinect [15]. It is used for rehabilitation of upper limb shoulder and elbow joint motor dysfunction in patients with cerebrovascular diseases. After the rehabilitation treatment of cerebrovascular patients for 2 weeks with this system, the FMA score and Wolf score of the patients were improved compared with those before the treatment, and the improvement was larger than that of the conventional treatment group. Kinect motion-sensing interaction technology is combined with VR technology, and Kinect sensor is used to replace many traditional complex and expensive sensors, so that patients can directly control objects in the virtual scene through the movement of their limbs, without the need to wear a sensor, so as not to increase the burden on the injured limbs of patients, so that they can better complete the rehabilitation training. It also dramatically reduces the cost of the device, making it easy to market in the community and even in the home. At the same time, this study focused on cerebrovascular disease, including stroke, abnormal intracranial aneurysm, intracranial vascular malformations, moyamoya disease. This study has children, youth stroke cases, which proved that somatosensory interaction mechanism of virtual reality technology is effective to upper limb dysfunction caused by different cerebrovascular disease, there was no evidence of different effects for different age groups of patients.

In this study, FMA-UE score, WMFT score and MBI score of patients in the control group and the treatment group after 4 weeks of treatment all increased significantly compared with those before treatment, and the virtual reality group showed more significant improvement compared with the control group. Cortical remodeling emphasizes the importance of repetition and task specificity [16]. Task-specific treatment is an important factor in functional regeneration of cortical regions responsible for related neural activity. Whether targeting a specific motion (such as elbow flexion) or a skill (such as self-grooming), specificity is a must [17]. In this study, virtual scenarios were simulated to provide patients with meaningful, specific, and repetitive training. Through learning and exercise rehabilitation, the maximum degree of neural remodeling could be promoted, thus promoting the effect of rehabilitation. This study provides a three-dimensional virtual environment in which patients play games in a rich and pleasant virtual environment provided by the system, providing patients with colorful visual stimuli. At the same time, when patients complete the task, there will be an encouraging voice to encourage patients to repeat the same movement. It has greatly increased their enthusiasm to participate in rehabilitation treatment. FMA score was established based on Brunnstrom's evaluation method, and was evaluated in turn according to the recovery process of hemiplegia. The rehabilitation game in this study was designed according to Brunnstrom's theory. In the food cutting game, the upper limb virtual knife is used to cut the food in the cupboard. In the shooting game, the upper limb is used to control the gun shooting, so as to suppress the abnormal flexure mode of the upper limb, induce the patients to change synnergy movement to isolated movement, and promote the patients to strengthen the normal movement mode. Maintain and expand the range of motion of the shoulder and elbow joint, improve the stability of the shoulder and elbow joint, and improve the ability of joint motion control. In this way, the recovery of upper limb motor function was promoted, and the FMA score of the patients was higher than before. In this study, through cutting food and shooting games, not only the movement of a single joint of the shoulder and elbow joints was trained, but also the combined movements of shoulder flexion, elbow extension, adduction and abduction of the affected side upper limb were trained in the form of games to promote the ability of the upper limb to reach and reach forward. Therefore, it can better improve the motor function of the upper limb of the affected side of the patient and significantly increase the WMFT score of the patient. In this study, the reasons for the improvement of MBI score in patients were considered as the improvement of upper limb function and task-specific training, which made it easier for patients to transfer the skills acquired in training to daily life.

\section{CONCLUSION}

The results of the study confirmed that the virtual reality technology based on Kinect was effective in 
the treatment of upper limb dysfunction in the convalescent patients of cerebrovascular disease, and it could partly replace the active rehabilitation training program of traditional operation treatment. However, this study has some limitations. The study requires patients to have better cognitive function, and the object of this study is the convalescent period of cerebrovascular disease. It requires patients to reach grade III and above in Brunnstrom stage of upper limb, which is not applicable to patients with severe upper limb dysfunction. And due to the poor accuracy of hand knuckle recognition of Kinect sensor, the treatment of finger dysfunction cannot be carried out in this study. This study can only carry out active rehabilitation training of upper limb movement, but cannot complete passive training. It is in the early research stage, and the follow-up data have not been collected and analyzed. The follow-up sample size should be increased for the experiment, and the patients should be followed up to observe the continuity of the efficacy.

\section{FUND PROJECT}

Guangzhou Health Science and Technology Project (Project Number: 20181A010070).

\section{CONFLICTS OF INTEREST}

The authors declare no conflicts of interest regarding the publication of this paper.

\section{REFERENCES}

1. Green, T.L. and King, K.M. (2010) Functional and Psychosocial Outcomes 1 Year after Mild Stroke. Journal of Stroke and Cerebrovascular Diseases, 19, 10-16. https://doi.org/10.1016/j.jstrokecerebrovasdis.2009.02.005

2. Roger, V.L., et al. (2011) Heart Disease and Stroke Statistics-2011 Update: A Report from the American Heart Association. Circulation, 123, e18-e209.

3. Turolla, A., Dam, M., Ventura, L., Tonin, P., Agostini, M., Zucconi, C., et al. (2013) Virtual Reality for the Rehabilitation of the Upper Limb Motor Function after Stroke: A Prospective Controlled Trial. Journal of NeuroEngineering and Rehabilitation, 10, 85. https://doi.org/10.1186/1743-0003-10-85

4. Ward, N.S., Kelly, K. and Brander, F. (2015) The Future of Stroke Rehabilitation: Upper Limb Recovery. Advances in Clinical Neuroscience \& Rehabilitation, 15, 6-9.

5. Deutsch, J.E. (2011) Using Virtual Reality to Improve Walking Post-Stroke: Translation to Individuals with Diabetes. Journal of Diabetes Science and Technology, 5, 309-314. https://doi.org/10.1177/193229681100500216

6. Maier, M., Rubio Ballester, B., Duff, A., et al. (2019) Effect of Specific over Nonspecific VR-Based Rehabilitation on Poststroke Motor Recovery: A Systematic Meta-Analysis. Neurorehabilitation and Neural Repair, 33, 112-129. https://doi.org/10.1177/1545968318820169

7. Xiao, X., Huang, D.F. and Liang, B. (2019) Effects of Virtual Reality Technique in Rehabilitation of Hemiplegic Upper Extremities Function in Stroke Patients. Chinese Journal of Rehabilitation Medicine, 34, 1049-1053.

8. (1996) Diagnosis Point of Cerebrovascular Diseases. Chinese Journal of Neurology, 29, 379-380.

9. Hatem, S.M., Saussez, G., della Faille, M., Prist, V., Zhang, X., Dispa, D. and Bleyenheuft, Y. (2016) Rehabilitation of Motor Function after Stroke: A Multiple Systematic Review Focused on Techniques to Stimulate Upper Extremity Recovery. Frontiers in Human Neuroscience, 10, 442. https://doi.org/10.3389/fnhum.2016.00442

10. Laver, K.E., Lange, B., George, S., Deutsch, J.E., Saposnik, G. and Crotty, M. (2017) Virtual Reality for Stroke Rehabilitation. Cochrane Database of Systematic Reviews, 11, CD008349. https://doi.org/10.1002/14651858.CD008349.pub4

11. Levin, M.F., Weiss, P.L. and Keshner, E.A. (2015) Emergence of Virtual Reality as a Tool for Upper Limb Rehabilitation: Incorporation of Motor Control and Motor Learning Principles. Physical Therapy, 95, 415-425. 
https://doi.org/10.2522/ptj.20130579

12. Cortés, C., Ardanza, A., Molina-Rueda, F., et al. (2014) Upper Limb Posture Estimation in Robotic and Virtual Reality-Based Rehabilitation. BioMed Research International, 2014, Article ID: 821908. https://doi.org/10.1155/2014/821908

13. Ustinova, K.I., et al. (2011) Development of a 3D Immersive Videogame to Improve Arm-Postural Coordination in Patients with TBI. Journal of NeuroEngineering and Rehabilitation, 8, 1. https://doi.org/10.1186/1743-0003-8-61

14. Merians, A.S., Fluet, G.G., Qiu, Q., et al. (2011) Robotically Facilitated Virtual Rehabilitation of Arm Transport Integrated with Finger Movement in Persons with Hemiparesis. Journal of NeuroEngineering and Rehabilitation, 8, 27. https://doi.org/10.1186/1743-0003-8-27

15. Qu, C., Dai, A.B., Guo, A.S., et al. (2015) Development and Application of a Kinect-Based Upper-Limb Rehabilitation System. Chinese Journal of Biomedical Engineering, 34, 607-612.

16. Johansson, B. (2011) Current Trends in Stroke Rehabilitation. A Review with Focus on Brain Plasticity. Acta Neurologica Scandinavica, 123, 147-159. https://doi.org/10.1111/j.1600-0404.2010.01417.x

17. Hallett, M. (2001) Plasticity of the Human Motor Cortex and Recovery from Stroke. Brain Research, 36, 169-174. https://doi.org/10.1016/S0165-0173(01)00092-3 